EPJ Web of Conferences 22, 00003 (2012)

DOI: $10.1051 /$ epjconf/20122200003

(C) Owned by the authors, published by EDP Sciences, 2012

\title{
Symmetry analysis of magnetic structures on the microscopic and macroscopic methods of E.F. BERTAUT
}

\author{
D. Fruchart ${ }^{\mathrm{a}}$
}

Institut Néel, CNRS et Université Joseph Fourier, BP. 166, 38042 Grenoble Cedex 9, France

\section{INTRODUCTION}

Magnetic long range ordering was questioned experimentally and theoretically, very early by Weiss [1], Dirac [2], Heisenberg [3], Van Vleck [4] and others, before the hypothesis of antiferromagnetism was developed by Néel [5], as well as before the hypothesis of Rutherford concerning [6] the existence of the neutron was confirmed by Chadwick [7]. As a revelation, the experimental proof of antiferromagnetic (AF) ordering in $\mathrm{MnO}$ demonstrated by Shull and Smart [8] enlightened the unique powerfulness of neutron scattering in asserting definitively the prediction of Néel. At that critical period, Bertaut emerged rapidly as one of the most prolific and brilliant solid state chemists and physicists aiming to relate systematically the physical properties of solid state materials to crystal structure symmetry.

\section{E. F. BERTAUT'S LIFE BEFORE ACADEMIC RESEARCH}

Erwin Lewy was born in a Jewish germanophile family on Feb. $9^{\text {th }} 1913$ in the small town of Leobschutz (now Gklubczyce, Poland), High Silesia. Excellent in music and mathematics, however he began lawyer studies in Breslau (Wroclaw). He red "Mein Kampf" and experienced the fascist climate developing at the period. After being injured by "Youth Nazis", in 1933 he decided to migrate to Paris then to Bordeaux (France) where he studied chemistry at the Ecole de Chimie de Bordeaux. Graduate Chemist Engineer, he got the French citizenship in 1936. He worked as a chemist at the company "La Cellulose du Pin" where, among several activities, he investigated hydrogenation processes under high pressure assisted by catalysts such as Raney nickel.

Most of his close family who had joined him in Bordeaux were arrested in 1940, then deported and exterminated in Auschwitz.

Early he engaged in the French army, and later when demobilised, an officer proposed him a new identity card with name Felix Bertaut. Working in Paris at the Service de Poudres in 1941-1942, Bertaut learned crystallography under the supervision of Prof. J.P. Matthieu. But, Prof. A. Kastler suggested him to leave Paris and he recommend to Prof L. Néel to host him in Grenoble, an unforgettable facility opening the exceptional career of Erwin Felix Lewy-Bertaut.

\section{E. F. BERTAUT'S TRIBUTE TO SOLID STATE CHEMISTRY AND PHYSICS}

Bertaut was soon introduced in the field of magnetic properties of materials since his thesis work [9] supervised by Néel has consisted mostly in the determination of the crystallite size in ferromagnetic compounds, a dimension parameter making sense for coercivity, as an extrinsic property of hard magnets. Immediately Bertaut has developed an original approach in computing the broaden profile

\footnotetext{
${ }^{a}$ e-mail: daniel.fruchart@grenoble.cnrs.fr
}

This is an Open Access article distributed under the terms of the Creative Commons Attribution-Noncommercial License 3.0, which permits unrestricted use, distribution, and reproduction in any noncommercial medium, provided the original work is properly cited. 


\section{EPJ Web of Conferences}

of the X-ray diffraction lines by Fourier's transform analysis, his method being more effective than the most referred by Warren and Averbach [10].

As said above, the same year, the pioneer neutron diffraction experiment by Shull and Smart [8] was published to confirm, with elegancy, the theoretical prediction of antiferromagnetic sublattices by Néel [5]. So in 1951 Bertaut moved first to USA, then in 1953 he was granted as Fullbright fellowship to spend a year at Brookhaven National Laboratory to study neutron scattering sciences. Two years after, Néel initiated the creation of the Centre d'Etudes Nucléaires de Grenoble (CENG later CEA-Grenoble) where two neutron research reactors Mélusine (up to 12 MW - 1958) and Siloé (up to 32 MW - 1962) were successively operated. Bertaut was in charge of their instrumental development. As well during this period, Bertaut started his intense continuous work on the symmetry analysis of magnetic structures. Those milestones of his long-term academic activity will be more detailed in the following parts of the present article.

Parallel to his main activity devoted to neutron diffraction and symmetry analysis of magnetic configurations, Bertaut continued using his impressive facilities for crystallography. First he built a new reciprocal space formalism to determine the electrostatic energy of crystalline systems [11]. The Bertaut's method was demonstrated to have a much better effective convergence than that of Ewald, early built on the basis of direct space operations [12]. During this period, another and important contribution of Bertaut to crystal structure determination was to build a general algebra of the structure factors for a given crystal accounting for the equivalent site positions. The linearised products built from these structure factors led him thus to determine statistics and extinctions rules [13]. His contribution to the so-called direct method for crystal structure determination, just proposed by e.g. Zachariasen [14] and Wilson [15], was based on the search for symmetry relationships existing between the structure factors. From this early and many following works as eminent crystallographer, Bertaut was invited to refund the basis of the International Tables for Crystallography in straight cooperation with Hahn [16].

All along his prolific career, systematically Bertaut felt immediately concerned for all aspects of solid state chemistry and solid state physics, promptly inciting researches on new materials, to use original synthesis methods (i.e. large single crystals, e.g. at LETI), to study novel phenomena in magnetism, superconductivity, metal-insulator transitions, X-ray magnetic scattering (experimental evidence in his laboratory by de Bergevin and Brunel [17]). His interests to experimental chemistry and crystallography concerned large panels of solid state compounds from alloys and intermetallics, hydrides, carbides, pnictides, chalcogenides, halides, complex oxides (e.g. the famous garnets, also many others with rare earth and actinides elements), borates, silicates, phosphates, etc.

To serve both his large experimental and conceptual scientific appetites, he actively promoted the development of novel techniques such as neutron time of flight, the use of polarised neutrons at Mélusine, in laboratory and under beam high pressure equipments, computer crystal structure determination in 1964 etc. In parallel, Bertaut engaged the premises of new large array instruments, directly participating to initiate, early 1963, the Institute Laue-Langevin erected in 1971. In 1981 he supported strongly the project SIREM (Source Intense de Rayonnement Electromagnétique), a proposal for a French synchrotron in Grenoble, a few time after, rescaled in the international proposal for the European Synchrotron Radiation Facilities (1984).

However during this period of intense multidisciplinary activities, symmetry analysis was his main guideline aiming to better understand a wide panel of solid state materials, from chemistry principles up to sophisticated instruments built to establish and finely detail the correlations and in the matter.

\section{BACKGROUND}

Magnetic energy, magnetic couplings, magnetic ordering, group theory representations, etc., are master expressions which were employed for different purposes, at different periods, for times used opposite to built various models, then admitted to converge for a unique point of view, thanks to well known pioneers in magnetism and crystallography, thanks to Bertaut due to its synthetic lecture and interpretation. 


\section{Contribution of Symmetries in Condensed Matter}

Early after the definition of the electron and orbital moments and the spectroscopic rules (Pauli principle, Hund's rules) leading to attribute a permanent magnetic moment to well defined classes of elements, thermal analysis of magnetism by Weiss opened the question of exchange forces [1] whereas Dirac [2] then Heisenberg [3] formulated the notion of exchange hamiltonian to describe a ferromagnetic material via a representative energy. This can be called the analytic or thermodynamic point of view on magnetic correlations and magnetic ordering stability. This results from the concept of molecular field used successfully by Néel to predict antiferromagnetism [5] and developed successively by Van Vleck [4], Kittel [18], Villain [19] etc. for more and more different cases up to non-collinear magnetic configurations.

On the other side, a fully mathematical analysis of symmetry was proposed by Herring, as a theory of group representations [20]. The Landau's school of phase transition aimed at describing magnetic space invariant expressions to be considered in a more generalised hamiltonian. Then Belov [21], Kovalev [22], Dzyaloshinski [23] opened the way of a crystallographic approach. They were followed in more synthetic analysis in terms of symmetry operators by Vonsovski and Turov [24], Moriya [25], Opechowski and Guccione [26], etc.

Since the hypothesis of neutron by Rutherford [6] that Chadwick confirmed experimentally [7], Shull and Smart have proven neutron scattering to be a unique tool to visualise magnetic material orderings (e.g. AF of MnO) [8]. Then, during the period 1953-1958, Bertaut as a fresh crystallographer has balanced very actively from experiments using neutron diffraction to build configuration models by using the two main approaches, and publishing first synthetic analyses in 1960-1969, then rationalised in several main papers [27].

\section{MICROSCOPIC VERSUS MACROSCOPIC ANALYSES OF BERTAUT}

\subsection{The microscopic method of Bertaut [28]}

Roughly between 1961 [29] and 1973 [28], Bertaut has developed successively more and more sophisticated mathematics enabling to determine all possible sets of moment configurations according to the local environment of a given magnetic atom $\mathbf{R}$, as the early basis of molecular field model expressed in the Hamiltonian of Eq. (I). The method accounts for the different Bravais lattices as $\mathbf{S}_{\mathbf{R}}$ carrying magnetic moments and then only the permutation group operators are involved and $J$ is a scalar.

$$
H=-2 \sum_{\mathbf{R}, \mathbf{R}^{\prime}} J_{\mathbf{R R}^{\prime}} \mathbf{S}_{\mathbf{R}} \mathbf{S}_{\mathbf{R}^{\prime}}
$$

To determine the equilibrium condition of $\mathbf{S}_{\mathbf{R}}$ "oscillating" moment at position $\mathbf{R}$ one differentiates (I) under the constraint $S_{\mathbf{R}}^{2}=$ constant:

$$
\hbar \frac{d \mathbf{S}_{\mathbf{R}}}{d t}=2 \sum_{\mathbf{R}^{\prime}} J_{\mathbf{R R}^{\prime}} \mathbf{S}_{\mathbf{R}^{\prime}} \wedge \mathbf{S}_{\mathbf{R}}
$$

At equilibrium $\left(d \mathbf{S}_{\mathbf{R}} / d t=\mathbf{0}\right), \mathbf{S}_{\mathbf{R}}$ must be parallel to the molecular field $\Sigma_{\mathbf{R}^{\prime}} J_{\mathbf{R}^{\prime}} \mathbf{S}_{\mathbf{R}^{\prime}}$ where parallelism condition corresponds to $\lambda_{\mathbf{R}} \sigma_{\mathbf{R}}=\Sigma_{\mathbf{R}^{\prime}} \boldsymbol{J}_{\left(\mathbf{R R}^{\prime}\right)} \boldsymbol{\sigma}_{\mathbf{R}^{\prime}}$ with $\boldsymbol{J}_{\left(\mathbf{R R}^{\prime}\right)}=S_{\mathbf{R}} J_{\mathbf{R}^{\prime}} S_{\mathbf{R}^{\prime}}$, the unit spin being defined as $\sigma=\mathbf{S} / S$. $\lambda_{\mathbf{R}}$ is a real number, as Bertaut said a Lagrange parameter, or the contribution of the $\mathbf{R}$ atom to the exchange energy via:

$$
H=-2 \sum_{\mathbf{R}} \lambda_{\mathbf{R}}
$$

$\lambda_{\mathbf{R}}$ and $H$ are invariant under the crystal symmetry. If one considers the different Bravais lattices $\mathbf{R}$ (now labeled $r$ with indices $i, j=1$ to $n), \lambda_{i} \sigma_{i}\left(\mathrm{r}_{i}\right)$ is multiplied by $\exp \left(i q \cdot \mathrm{r}_{i}\right)$, and summing on all $\mathbf{r}_{i}$ of the 
$i$ lattice leads defining:

$$
\lambda_{i} \mathbf{T}_{i}(\mathbf{q})=\sum_{j} \xi_{i j}(\mathbf{q}) \cdot \mathbf{T}_{j}(\mathbf{q})
$$

where $\mathbf{q}$ is the propagation vector. As $\boldsymbol{J}_{\left(\mathbf{r}_{i} \mathbf{r}_{j}\right)}$ only depends on the distance $\left|\mathbf{r}_{i}-\mathbf{r}_{j}\right|$, for the whole crystal of $\mathcal{N}$ unit cells, one gets $n$ equations like:

$$
\mathcal{N} \mathbf{T}_{i}(\mathbf{q})=\sum_{\mathbf{r}_{i}} \sigma_{i}\left(\mathbf{r}_{i}\right) \exp \left(i \mathbf{q} \cdot \mathbf{r}_{i}\right) \quad \text { and } \quad \xi_{i j}(\mathbf{q})=\sum_{\mathbf{r}_{j}} J_{\left(\mathbf{r}_{i} \mathbf{r}_{j}\right)} \exp \left[i \mathbf{q} \cdot\left(\mathbf{r}_{i}-\mathbf{r}_{j}\right)\right]
$$

with $\mathbf{T}_{i}(\mathbf{q})$ the Fourier transform of spin $\boldsymbol{\sigma}_{i}\left(\mathbf{r}_{i}\right)$ and $\xi_{i j}(\mathbf{q})$ the interaction matrix. Then one fixes $\mathbf{r}_{i}$ and sums on all equivalent $\mathbf{r}_{j}$ around the $\mathbf{r}_{i}$. This can be written in the form:

$$
[\xi(\mathbf{q})-(\lambda)] \cdot \mathbf{T}(\mathbf{q})=0
$$

where $\xi(\mathbf{q})$ is a hermitic matrix of order $n,(\lambda)$ being a diagonal matrix and $\mathbf{T}(\mathbf{q})$ a vector of order $n$. This column vector has components $\mathbf{T}_{i}(\mathbf{q})$ which are Fourier transforms of real spin components $\boldsymbol{\sigma}_{i}=\boldsymbol{\sigma}\left(\mathbf{r}_{i}\right)$. So one can write:

$$
\sigma\left(\mathbf{r}_{i}\right)=\sum_{\mathbf{q}} \mathbf{T}_{i}(\mathbf{q}) \cdot \exp \left(-i \mathbf{q} \cdot \mathbf{r}_{i}\right)
$$

This is the basis of a first series of analyses written by Bertaut thus allowing to determine and justify collinear and non-collinear magnetic moment configurations. Bertaut also proposed another writing and easiest to use formalism.

Since the $\xi(\mathbf{q})$ matrix is atom coordinate depending, the new $\eta(\mathbf{q})$ defined by $\xi_{i j}(\mathbf{q})=$ $\eta_{i j}(\mathbf{q}) \exp \left[i \mathbf{q} .\left(\mathbf{r}_{i 0}-\mathbf{r}_{j 0}\right)\right]$ only depends on the Bravais translations $\left(\mathbf{r}_{i 0}\right.$ and $\mathbf{r}_{j 0}$ being the origins of the Bravais lattices). New eigenvectors are defined $\mathbf{Q}_{j}(\mathbf{q})=\mathbf{T}_{j}(\mathbf{q}) \exp \left(-i \mathbf{q} \cdot \mathbf{r}_{j 0}\right)$ leading to a similar form as (VI) but a simplest interaction matrix:

$$
[\eta(\mathbf{q})-(\lambda)] \cdot \mathbf{Q}(\mathbf{q})=0
$$

The real magnetic components can be developed as (assuming only one propagation vector $\mathbf{q}$ ):

$$
\sigma\left(\mathbf{r}_{j 0}\right)=\mathbf{Q}_{j}(\mathbf{q})+\mathbf{Q}_{j}^{*}(\mathbf{q}) \quad \text { with } \quad \mathbf{Q}_{j}^{*}(\mathbf{q})=\mathbf{Q}_{j}(-\mathbf{q})
$$

If one considers general solutions such as $\mathbf{Q}_{j}(\mathbf{q})=\frac{1}{2}(\mathbf{u}+i \mathbf{v}) Q_{j}$ where $Q_{j}$ is a phase factor in form $Q_{j}=\exp \left(-i \psi_{j}\right)$

The magnetic moment can be written as follows:

$$
\mathbf{S}\left(\mathbf{r}_{j 0}\right)=S_{j}\left(\mathbf{u} \cos \psi_{j}+\mathbf{v} \sin \psi_{j}\right)
$$

\subsection{The macroscopic method of Bertaut [30]}

Here the whole set of symmetry operators of a space group is considered and $\boldsymbol{J}$ can have the form of a 2-dimension tensor. Again anisotropy operators can be considered. Magnetic moments are effectively treated as axial vectors, for which Belov [21] and Kovalev [22] have already defined the effects of operators and anti-operators, depending on the time reversal operator effect. This method likes to be rigorously absolute as based on the full symmetry of a considered space group, which symmetry must be well identified first. The method is better explicitly called "theory of space group representations" as for the most extended version [30].

Given a space group $G$, a representation $\Gamma(A)$ of dimension $d$ of this space group is a set of square matrices $d \times d$ (one for each symmetry operator $A$ ) which is isomorphic to $G$. The table of characters $\chi(A)$ for a representation is defined by the traces of the $A$ matrices, a quantity which is invariant for all equivalent representations and is used in fine to build the basis vectors of magnetic configurations. The 


\section{Contribution of Symmetries in Condensed Matter}

purpose of the reduction procedure of a representation $\Gamma(A)$ is to find a new basis allowing to form a direct sum of diagonal matrices representation such as:

$$
\Gamma(A)=\Gamma_{1}(A) \oplus \Gamma_{2}(A) \oplus \Gamma_{3}(A) \ldots
$$

When it becomes impossible to operate a full reduction (non-diagonal matrix block, indices $(i, j)$ ), the processed representation is said irreducible, the corresponding sum (XI) ending as a set of $\Gamma_{v}(A)$, $d_{v}$ being the multiplicity of identical sub-matrices. Then building the table of characters $\chi_{v}\left(A_{n}\right)$ for the $v$ blocks of irreversibility and the $\mathrm{n}$ symmetry operators $A_{n}$ of the group $G$ allows to define the number and the dimension of the basis vectors. The corresponding magnetic configurations are formed from invariant linear combinations (in $G$ ) of moment components $S_{\alpha}^{m}$ where $\alpha=x, y, z$ and $m$ numbers the multiplicity of a magnetic site position.

To derive the definitive set of the basis vectors $\mathbf{V}^{n}$, and then to build the Heisenberg type hamiltonian of order 2 in terms of eigenvector contributions, one has to use the projection operator technique ( $R_{A}$ being the matrices of the full space group representation):

$$
\mathbf{V}_{i j}^{n}=\frac{d_{\nu}}{g} \sum_{R_{A}} \Gamma_{i j}^{\nu}\left(R_{A}\right)^{*} \cdot R_{A} S_{\alpha}^{m}
$$

with $g$ the order of the group. As detailed in those following courses, expressly dedicated to the theory of space group representations, the here above description would like to serve only as an elementary report of the succession of algebraic mechanisms to be considered for. In fact, application of the theory of space group representations is more complicated owing to different criteria: for instance, the $G$ group might be symmorphic or not; the magnetic cell may coincide with the structural cell or not; in the latter case, the propagation vector $\mathbf{k}(\mathbf{q}=2 \pi \mathbf{k})$ is non-zero and might be commensurate, incommensurate or join the origin to a point of the Brillouin zone surface (the little group associated to the propagation vector $G_{0 \mathbf{k}}$ must then be considered instead of the point group $G_{0}$ ); this might lead to loaded representations; the magnetic structure might be described by several propagation vectors; if $G$ allows for more than one equivalent propagation vector (belonging to $\mathbf{k}^{*}$, the star of $\mathbf{k}$ ) this might lead to a possible mixture of elementary eigenvectors. If the phase transition is first order instead of second order (in which case the magnetic structure is described with a single irreducible representation), then several irreducible representations might be mixed, although not necessarily. Besides, through additional magnetoelectric or magnetoelastic couplings, there might be a lowering of the crystal symmetry associated to the magnetic ordering. So, lowering the G space group symmetry is a procedure that can be considered when considering the full symmetry of the starting space group does not lead to formulate eigenvectors in agreement with the neutron diffraction data.

\subsection{On two examples}

\subsubsection{The Phase Comparison Method: $\mathrm{RMn}_{2} \mathrm{O}_{5}$, where $\mathrm{R}=\mathrm{Y}$ or a Rare Earth element}

Bertaut presented in this example a simple method which does not need explicit knowledge of the eigenvectors and eigenvalues derived in the Microscopic approach but uses the fact that the linear equations belonging to the same eigenvalue can be made either identical or conjugate [31]. The space group Pbam of this series of oxides allows localising the cations in specific Wyckoff positions ${ }^{1}$ :

$$
\begin{array}{ll}
\mathrm{Mn}^{2+} \text { in site } 4 g\left(\mathrm{Mn}_{\mathrm{I}}\right) & \\
\mathrm{x}, \mathrm{y}, \mathrm{O}(1) ; & -\mathrm{x},-\mathrm{y}, 0(2) ; \\
\frac{1}{2}-\mathrm{x}, \frac{1}{2}+\mathrm{y}, \mathrm{O}(3) ; & \frac{1}{2}+\mathrm{x}, \frac{1}{2}-\mathrm{y}, 0 \text { (4) }
\end{array}
$$

${ }^{1}$ For a reminder on crystallography, see chapter by Grenier and Ballou. 


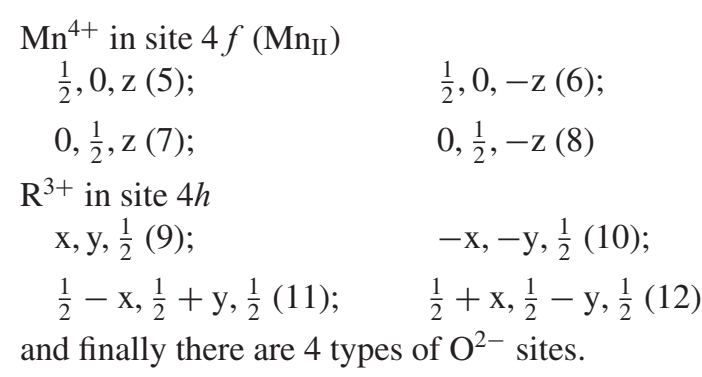

For $\mathrm{RMn}_{2} \mathrm{O}_{5}$, the propagation vector derived from low temperature neutron diffraction patterns is $\mathbf{q}=2 \pi \cdot\left(\frac{1}{2} \mathbf{a}^{*}+\tau \mathbf{c}^{*}\right)$.

First let us consider $\mathrm{YMn}_{2} \mathrm{O}_{5}$ : since $\mathrm{Y}$ does not carry any magnetic moment, according to (VIII), the $\eta(\mathrm{Mn})$ magnetic interaction is a $8 \times 8$ matrix.

$$
\eta(\mathrm{Mn})=\left(\begin{array}{cc}
\eta_{\mathrm{I}} & \eta_{\mathrm{I}-\mathrm{II}} \\
\eta_{\mathrm{I}-\mathrm{II}} & \eta_{\mathrm{II}}
\end{array}\right)
$$

System $M n_{I}$ : One can derive phase relationships, with $\psi_{i}$ the phase angles defined part 4.1, as:

$$
\psi_{4}-\psi_{3}=\psi_{1}-\psi_{2}+2 \pi . m \text { and } \psi_{1}-\psi_{3}=\psi_{4}-\psi_{2}+2 \pi . n
$$

where $\mathrm{m}$ and $\mathrm{n}$ are integers. Solutions are given by:

$$
\operatorname{tg} 2 \psi_{1}=-\boldsymbol{J}_{1}(1-3) / \boldsymbol{J}_{1}(1-2)
$$

System $M n_{I I}$ : No phase relationship can be established between moments onto sites 5 and 7 !

This indetermination can be lifted from the study of the fully $\mathrm{Mn}_{\mathrm{I}}-\mathrm{Mn}_{\mathrm{II}}$ coupled system:

$$
\begin{aligned}
& \boldsymbol{\sigma}_{1}=\mathbf{u} \cos \psi_{\mathrm{I}}+\mathbf{v} \sin \psi_{\mathrm{I}} \\
& \boldsymbol{\sigma}_{2}=-\mathbf{u} \cos \psi_{\mathrm{I}}+\mathbf{v} \sin \psi_{\mathrm{I}} \\
& \boldsymbol{\sigma}_{3}=\mathbf{u} \sin \psi_{\mathrm{I}}+\mathbf{v} \cos \psi_{\mathrm{I}} \\
& \boldsymbol{\sigma}_{4}=\mathbf{u} \sin \psi_{\mathrm{I}}-\mathbf{v} \cos \psi_{\mathrm{I}} \\
& \boldsymbol{\sigma}_{5}=\mathbf{u} \cos \psi_{\mathrm{II}}+\mathbf{v} \sin \psi_{\mathrm{II}} \\
& \boldsymbol{\sigma}_{6}=\mathbf{u} \cos \psi_{\mathrm{II}}-\mathbf{v} \sin \psi_{\mathrm{II}} \\
& \boldsymbol{\sigma}_{7}=-\mathbf{u} \sin \psi_{\mathrm{II}}+\mathbf{v} \cos \psi_{\mathrm{II}} \\
& \boldsymbol{\sigma}_{8}=\mathbf{u} \sin \psi_{\mathrm{II}}+\mathbf{v} \cos \psi_{\mathrm{II}}
\end{aligned}
$$

where $\mathbf{u}$ and $\mathbf{v}$ are two orthogonal unit vectors of the $(x, y)$ plane. Please notice the orthogonal directions of the 1 and 4 (2 and 3) magnetic moments as well as of the 5 and 7 (6 and 8) magnetic moments corresponding to rise of un-determination as remarked above. All details of the magnetic structure can be found in ref. [32].

System $\boldsymbol{R}^{3+}$ : For the magnetic rare-earth atoms, analysis of the $\eta(\mathrm{R})$ interaction matrix leads to build relationships similar to (XIV). However the $\mathrm{R}^{3+}$ sites are submitted to strong crystal field effects with an in-plane anisotropy direction along [210] for the $R_{9}$ and $R_{10}$ sites and along [210] for the $R_{11}$ and $\mathrm{R}_{12}$ sites, both latter being much marked owing to different $\mathrm{O}^{-}$neighbouring. Strong anisotropy effect results in a marked deviation from collinearity and in an oscillating structure along $c$ of the R magnetic moment system. The total interaction matrix allows to describe the magnetic moment arrangement, e.g. of the $\mathrm{Mn}_{\mathrm{II}}^{4+}$ system, which obeys the orthogonal arrangement issued from (XVI) before the magnetic ordering of the $\mathrm{R}^{3+}$ sublattice, but is coupled via new phase relationships at low temperature when 
the $\mathrm{R}^{3+}$ moments order, as: $\psi_{\mathrm{II}},-\psi_{\mathrm{II}}, \theta-\psi_{\mathrm{II}}, \theta+\psi_{\mathrm{II}}$ for $\mathrm{Mn}_{\mathrm{II}}$ sites 5 to 8 at $-z, z, 1-z$ and $1+z$ respectively, $\theta$ being introduced to account for the Mn magnetic coupling with the $\mathrm{R}^{3+}$ moments.

The stability of these complex non-commensurate magnetic structures was confirmed by solving the determinant of second order derivations (see XVII) of the global interaction Hamiltonian $W$ versus three phases $\psi_{\mathrm{I}}, \psi_{\mathrm{II}}$ and $\theta$ :

$$
\Delta=\left|\begin{array}{ccc}
\frac{\partial^{2} W}{\partial \psi_{\mathrm{I}}^{2}} & \frac{\partial^{2} W}{\partial \psi_{\mathrm{I}} \partial \psi_{\mathrm{II}}} & \frac{\partial^{2} W}{\partial \psi_{\mathrm{I}} \partial \theta} \\
\frac{\partial^{2} W}{\partial \psi_{\mathrm{II}} \partial \psi_{\mathrm{I}}} & \frac{\partial^{2} W}{\partial \psi_{\mathrm{II}}^{2}} & \frac{\partial^{2} W}{\partial \psi_{\mathrm{II}} \partial \theta} \\
\frac{\partial^{2} W}{\partial \theta \partial \psi_{\mathrm{I}}} & \frac{\partial^{2} W}{\partial \theta \partial \psi_{\mathrm{II}}} & \frac{\partial^{2} W}{\partial \theta^{2}}
\end{array}\right|
$$

Therefore, the application of the Microscopic method allows justifying:

(i) the orthogonal couplings found experimentally between the magnetic moments of the two types of Mn sublattices when there is no ordered magnetic moment onto the $\mathrm{R}^{3+}$ sites $(\mathrm{R}=\mathrm{Y}$ or for the high temperature magnetic structure);

(ii) the low temperature long range oscillating magnetic structure, when $\mathrm{R}^{3+}$ carry an ordered magnetic $4 \mathrm{f}$ moment, moreover submitted to a crystal electric field producing a strong magnetic anisotropy.

In order to achieve a complete understanding of these particularly extended applications of the microscopic "phase comparison" method, please refer to ref. [31].

\subsubsection{The Macroscopic or Group Theory Method: $\mathrm{Mn}_{3} \mathrm{GaC}$}

The compound $\mathrm{Mn}_{3} \mathrm{GaC}$ is cubic (space group $\operatorname{Pm} \overline{3} m$, with $\mathrm{Ga}$ in site $1 a, \mathrm{C}$ in site $1 b$ and $\mathrm{Mn}$ in site $3 c$ ). It is antiferromagnetic (AF) below $T_{\mathrm{AF}} \sim 170 \mathrm{~K}$, then ferromagnetic (F) up to $T_{\mathrm{C}} \sim 240 \mathrm{~K}$, and finally paramagnetic at higher temperatures. Low temperature neutron diffraction patterns indicate the antiferromagnetic propagation vector $\mathbf{k}=\left(\frac{1}{2}, \frac{1}{2}, \frac{1}{2}\right)$ [33].

The analysis of the reduction conditions of the $\Gamma(G)$ complete transformation matrix and the search of the corresponding eigenvectors leads to its decomposition into irreducible blocks such as:

$$
\Gamma=A_{2 g} \oplus E_{g} \oplus T_{1 g} \oplus T_{2 g}
$$

Using the conventional spectroscopic notations, the four blocks form respectively $1\left(A_{2 g}\right), 2\left(E_{g}\right)$ and two $3\left(T_{1 g}\right.$ and $\left.T_{2 g}\right)$ dimensional systems. The corresponding eigenvectors are:

$$
\begin{aligned}
& \mathbf{V}\left(A_{2 g}\right)=\boldsymbol{\sigma}_{1 x}+\boldsymbol{\sigma}_{2 y}+\boldsymbol{\sigma}_{3 z} \\
& \mathbf{V}\left(E_{g}\right)=\left(\begin{array}{l}
\boldsymbol{\sigma}_{1 x}+\varepsilon \boldsymbol{\sigma}_{2 y}+\varepsilon^{*} \boldsymbol{\sigma}_{3 z} \\
\boldsymbol{\sigma}_{3 z}+\varepsilon \boldsymbol{\sigma}_{1 x}+\varepsilon^{*} \boldsymbol{\sigma}_{2 y}
\end{array}\right) \\
& \mathbf{V}\left(T_{1 g}\right)=\left(\begin{array}{l}
\boldsymbol{\sigma}_{3 y}+\boldsymbol{\sigma}_{2 z} \\
\boldsymbol{\sigma}_{1 z}+\boldsymbol{\sigma}_{3 x} \\
\boldsymbol{\sigma}_{2 x}+\boldsymbol{\sigma}_{1 y}
\end{array}\right) \quad \mathbf{V}\left(T_{2 g}\right)=\left(\begin{array}{l}
\boldsymbol{\sigma}_{3 y}-\boldsymbol{\sigma}_{2 z} \\
\boldsymbol{\sigma}_{1 z}-\boldsymbol{\sigma}_{3 x} \\
\boldsymbol{\sigma}_{2 x}-\boldsymbol{\sigma}_{1 y}
\end{array}\right)
\end{aligned}
$$




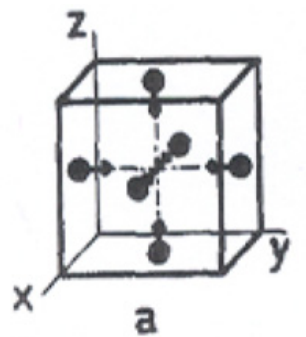

a

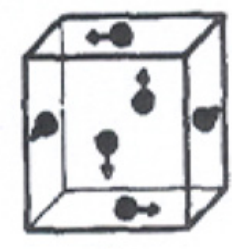

b

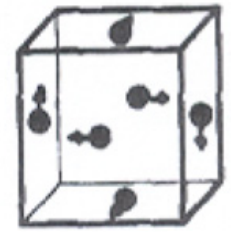

C

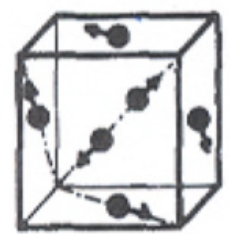

d

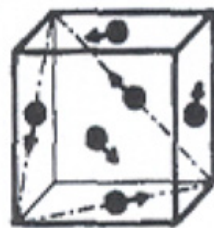

e

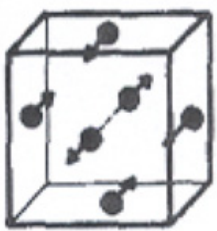

f

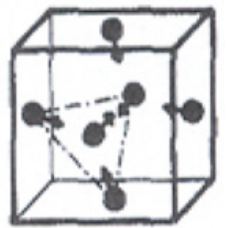

g

Figure 1. Antiferromagnetic modes than can be built from the representation theory analysis.

Pure modes with equal Mn moments can be successively derived as:

$$
\begin{aligned}
A_{2 g}: \boldsymbol{\sigma}_{1 x} & =\sigma_{2 y}=\sigma_{3 z} \neq 0 \\
T_{1 g}: \sigma_{1 x} & =\sigma_{2 y}=\sigma_{3 z}=0 \\
\sigma_{3 y} & =\sigma_{2 z}=\sigma_{1 z}=\sigma_{3 x}=\sigma_{2 x}=\sigma_{1 y} \neq 0 \\
T_{2 g}: \sigma_{1 x} & =\sigma_{2 y}=\sigma_{3 z}=0 \\
\sigma_{3 y} & =-\sigma_{2 z}=\sigma_{1 z}=-\sigma_{3 x}=\sigma_{2 x}=-\sigma_{1 y} \neq 0
\end{aligned}
$$

$E_{g}$ does not allow building an equal moments mode, as that experimentally determined.

To the magnetic arrangements that can be built, as shown in Fig. 1 (modes a to g), from the here above given pure modes (and their linear combination with respect to equal Mn moments), corresponds the same and unique value of the exchange energy when considering a Heisenberg type interaction hamiltonian of order 2 in moment components. Only some additional anisotropic terms should lead to different solutions. So, a question was: Why is the [111]-antiferromagnetic mode found experimentally stable by neutron diffraction? This magnetic arrangement corresponds to a stacking along the [111] direction of ferromagnetic planes of alternating sign, the moment direction being along the [111] direction.

Then lowering of the crystal symmetry was then critically analysed with respect to $\mathbf{k}=\left(\frac{1}{2}, \frac{1}{2}, \frac{1}{2}\right)$. The possible modes are the following: the mode a occurs in the identity representation $A_{1 g}$ of $P 23$ or $P m 3$ and also in the $A_{2 g}$ irreducible representation of $P 432, P \overline{4} 3 m, R 3 m, P m 3 m, R \overline{3} m$. The modes $\mathbf{b}$ and $\mathbf{c}$ occur in $T_{2 g}$ of $P 23$ and $P m 3$. The mode $\mathbf{d}$ is the sum of the moment configuration $\mathbf{b}$ and $\mathbf{c}$ and occurs in $T_{1 g}$ of $P 432, P \overline{4} 3 m, P m 3 m$. It occurs as well in $A_{2 g}$ of the rhombohedral groups $R 3 m$ and $R \overline{3} m$. The mode $\mathbf{e}$ is the difference of moment configuration $\mathbf{b}$ and $\mathbf{c}$ and occurs in $\mathrm{T}_{2 g}$ of $P 432, P \overline{4} 3 m, P m 3 m$ and also in $A_{1 g}$ of the rhombohedral group $R 3 m$ an $R \overline{3} \mathrm{~m}$. The mode $\mathbf{f}$ is the sum of moment configuration $\mathbf{a}+\mathbf{b}+\mathbf{c}(=\mathbf{a}+\mathbf{d})$ belonging to different representations in the cubic groups, and $\mathbf{a}+\mathbf{d}$ belonging to the same representation $A_{2 g}$ in $R 3 m$ and $R \overline{3} \mathrm{~m}$. The mode $\mathbf{g}$ is a triangular arrangement (Yafet-Kittel 


\section{Contribution of Symmetries in Condensed Matter}

type) in the (111) plane and belongs to the representation $E_{g}$ of the rhombohedral groups $R 3 m$ and $R \overline{3} m$. The simple ferromagnetic (111) plane belonging to $E_{g}$ of $R 3 m$ and $R \overline{3} m$ is not represented here.

As discussed here above, it comes that none of the cubic sub-groups leads to develop new noncollinear antiferromagnetic configurations than those already built from the Pm $3 m$ symmetry. The immediately first non-cubic group with the highest symmetry is the rhombohedral one $R \overline{3} m$ leading to:

$$
\Gamma(G)=A_{1} \oplus 2 A_{2} \oplus 3 E
$$

with configuration vectors:

$$
\begin{aligned}
& \mathbf{V}\left(A_{1}\right)=\boldsymbol{\sigma}_{1 y}-\boldsymbol{\sigma}_{1 z}+\boldsymbol{\sigma}_{2 z}-\boldsymbol{\sigma}_{2 x}+\boldsymbol{\sigma}_{3 x}-\boldsymbol{\sigma}_{3 y} \\
& \mathbf{V}_{1}\left(A_{2}\right)=\boldsymbol{\sigma}_{1 x}+\boldsymbol{\sigma}_{2 y}+\boldsymbol{\sigma}_{3 z} \\
& \mathbf{V}_{2}\left(A_{2}\right)=\boldsymbol{\sigma}_{1 y}+\boldsymbol{\sigma}_{1 z}+\boldsymbol{\sigma}_{2 z}+\boldsymbol{\sigma}_{2 x}+\boldsymbol{\sigma}_{3 x}+\sigma_{3 y}
\end{aligned}
$$

Combination of $\mathbf{V}_{1}\left(A_{2}\right)$ and $\mathbf{V}_{2}\left(A_{2}\right)$ belonging to the same irreducible representation $\mathrm{A}_{2}$ leads exactly to the collinear configuration directed along [111] as experimentally deduced from neutron diffraction data.

As a matter of proof:

1 - After this analysis, a very precise X-ray diffraction experiment was conducted with $\lambda_{K_{\alpha 1}(\mathrm{Cr})}$ to realise the highest resolution conditions. Actually, a weak rhombohedral distortion of about 89.7 degrees was evidenced, occurring down to $T_{\mathrm{AF}}$, thus confirming the direction [111] as the easy axis of the antiferromagnetic magnetic structure [33].

2 - Back to group theory considerations, it is worth noting that the antiferromagnetic mode can be generated only from different irreducible representations [34] when starting from the cubic space group $P m 3 m$. Since a 3D irreducible representation is concerned, as the considered sub-group contains the identity representation, it means that, at $T_{\mathrm{AF}}$, the transition cannot be of second order type. Actually, it is a first order transition, with a clear hysteresis in the experimental magnetisation traces and a net change in the Mn magnetic moment from $1.8 \mu_{\mathrm{B}}$ (AF phase) to $1.2 \mu_{\mathrm{B}}$ (F phase). Moreover, recent measurements by X-Ray Magnetic Circular Dichroism [35] have confirmed a strong magnetocaloric effect at $T_{\mathrm{AF}}$ to be related to the total entropy change from the magnetic (AF $\leftrightarrow \mathrm{F}$ ordering), electronic $\left(1.8 \leftrightarrow 1.2 \mu_{\mathrm{B}}\right)$ and a lattice contributions $(P m 3 m \leftrightarrow R \overline{3} m)$.

The theory of space group representations as developed by Bertaut revealed to be a powerful tool, not only to determine univocally the magnetic configuration according to the space group symmetry operators, but also to account for additional effects influencing the magnetic ordering (e.g. anisotropy tensors), as taught in specific papers (and herein references) of E. F. Bertaut [30, 36].

\section{CONCLUSION}

At first, Bertaut seemed fully convinced by the Microscopic point of view: “... they search, using group theory to establish a basis of irreducible representations allowing them to built a phenomenological Hamiltonian, which is invariant under the symmetry group operators. Our Method is Microscopic since it accounts for elementary operations. In our opinion, it is more powerful, ... since it allows to distinguish different modes from stability conditions, and to determine possible ranges of effectiveness for exchange integrals" (in French in [29]).

But rapidly he understood that according to Dirac, mathematics is a unique key for physics ("this results is too beautiful to be false, it is more important to have beauty in one's equation than to have them fit experiment" [37]). Its growing interest in the Macroscopic method was also supported by the impressive work realised by his groups, both at Laboratoire de Cristallographie du CNRS and at Laboratoire de Diffraction Neutronique du Centre d'Etudes Nucléaires de Grenoble (Commissariat à l'Energie Atomique). 
The original intuition of Bertaut was to understand early and to propose that the Microscopic and Macroscopic analyses form almost equivalent methods, both taking into account the symmetries of the system and based on a Heisenberg type of hamitonian as derived from the molecular field theory (for $1^{\text {st }}$ order developments) $[36,38,39]$.

Furthermore, Bertaut has participated to demonstrate that the theory of representations was definitively more complete than the earlier analysis via magnetic group considerations (direct and anti-operators [40]). The later one led to develop 1D eigen vectors only (those corresponding to 1D representations) with no mixed vectors (as from different irreducible representations) contrarily to the former one from where $2 \mathrm{D}$ and $3 \mathrm{D}$ eigen vectors could describe more complex magnetic arrangements, e.g. non collinear Yafet-Kittel type magnetic structures...

The Microscopic method is interesting since it allows building easily magnetic phase diagrams with respect to the relative values attributed to the exchange forces, furthermore accounting for the periodicity of the magnetic structure.

However, using the Macroscopic method (theory of representation) allows easily justifying symmetric or anti-symmetric couplings, thus accounting for parameters related for instance to magnetocrystalline anisotropy or spin-orbit couplings.

In fact, Bertaut has, in parallel to others, extended successfully both approaches to other types of solid state phenomena e.g. magnetoelectrics, ferroelectrics [41], magnetostrictive effects...

\section{References}

[1] P. Weiss, J. Phys., 6 (1907) 667.

[2] P. A. M. Dirac, Procc. of the Royal Soc. London, A112, 762, 1 (1928) 661.

[3] W. Heisenberg, Zeits. f. Physik, 38, 6-7 (1926) 411 and Zeits. f. Physik, 49 (1928) 619.

[4] J. Van Vleck, Nature, May 7 (1926); Phys. Rev., 29 (1927) 727 and Phys. Rev., 31 (1928) 587.

[5] L. Néel, Ann. Phys., 17 (1932) 5.

[6] H. Rutherford, Procc of the Royal Soc. London, A, 97 (1920) 374.

[7] J. Chadwick, Proc. of the Royal Soc. London, A, 136 (1932) 692.

[8] C. G. Shull and J. S. Smart, Phys Rev., 76 (1949) 1256.

[9] E. F. Bertaut, Thèse de l'Université, Grenoble (1949).

[10] B. E. Warren and B.L. Averbach, J. Appl. Phys., 21 (1950) 595.

[11] E. F. Bertaut, J. de Physique et le Radium, 13 (1952) 499.

[12] P. P. Ewald, Ann. Physik, 64 (1921) 253.

[13] E. F. Bertaut, Acta Cryst., 9 (1956) 769.

[14] F. W. H. Zachariasen, J. Chem. Phys. 3 (1935) 197 and Acta Cryst., 5 (1952) 68.

[15] A. J. C. Wilson, Nature 150 (1942) 151 and Acta Cryst. 2 (1949) 318.

[16] E. F Bertaut, "International Tables for Crystallography” Volume A - Space Group Symmetry, Ed. O. Hahn, The Intern. Union of Cryst., D. Reidel Pub., Dordrecht, 1985.

[17] F. de Bergevin and M. Brunel, Acta Cryst., A37 (1981) 314.

[18] Ch. Kittel, Phys. Rev., 70 (1946) 965.

[19] J. Villain, J. Phys. Chem. Solids, 11 (1959) 303.

[20] C. Herring, J. Franklin Institute, 223, 6 (1946) 525.

[21] N. V. Belov, Leeds Phil. and Literary. Soc. (1957).

[22] O. V. Kovalev, Kiev Izd-vo Academik nauk Ukr. (1961).

[23] I. E. Dzyaloshinski, Soviet Physics, JETP, 5 (1957) 1259; J. Expt. Theor. Phys. 32 (1957) 1547 and Soviet Phys. JETP, 10 (1959) 628.

[24] S. V. Vonsovskii and E. A. Turov, J. Appl. Phys., 29 (1959) 9.

[25] T. Moriya, Phys. Rev. 120 (1960) 91 and in “Magnetism”, Vol I, G. T. Rado and M. Suhl Ed., N. Y. Academic, 1963. 
[26] W. Opechowski and R. Guccione, in "Magnetism", Vol IIA, G.T. Rado and M. Suhl Ed., N. Y. Academic, 1965.

[27] E. F. Bertaut, J. Phys. Chem. Solids, 21 3-4 (1961) 256; J. de Physique et le Radium 22 (1961) 839 and in "Magnetism", Vol III, G. T. Rado and M. Suhl Ed., N.Y. Academic, 1963.

[28] E. F. Bertaut, C.R. Acad. Sc. Paris, B 277 (1973) 583.

[29] E. F. Bertaut, J. Phys. \& Chem. Sol. 24 (1961) 256.

[30] E. F. Bertaut, Acta Cryst. A24 (1968) 217.

[31] E. F. Bertaut J. de Phys., 35 (1974) 659.

[32] G. Buisson, Phys. Stat. Sol., 17 (1973) 191.

[33] D. Fruchart, E. F Bertaut, F. Sayetat, M. Nasr-Eddine, R. Fruchart and J. P. Sénateur, Solid State Comm., 8 (1970) 91.

[34] D. Fruchart and E. F. Bertaut, J. Phys. Soc. Jpn, 44 (1978) 781.

[35] L. H. Lewis, D. Yoder, A. R. Maudenbaugh, D. Fisher and M-H Yu, J. Phys. Cond. Matter, 18, 5 (2006) 1677.

[36] E. F. Bertaut, J. Magn. Magn. Mater., 24, 267 (1981) 76.

[37] P. A. M. Dirac, Scientific American, 208 (1963) 5.

[38] E. F. Bertaut, J. Phys. Chem. Solids, 39 (1978) 97.

[39] E. F. Bertaut,, J. Phys. Chem Solids, 39 (1978) 1331.

[40] A. V. Shubnikov and N. V. Belov, in "Symmetry in Science and Art", V. A. Kopsik Ed., Pergammon Press, London, 1974.

[41] E. F. Bertaut and M. Mercier, Mat. Res. Bull., 6 (1971) 907. 